\title{
Madeleine de Scudéry: une femme de lettres au XVII siècle, études réunies par Delphine Denis et Anne Elisabeth Spica
}

\section{Benedetta Papasogli}

\section{(2) OpenEdition}

\section{Journals}

\section{Edizione digitale}

URL: https://journals.openedition.org/studifrancesi/40577

DOI: $10.4000 /$ studifrancesi. 40577

ISSN: 2421-5856

\section{Editore}

Rosenberg \& Sellier

\section{Edizione cartacea}

Data di pubblicazione: 1 juillet 2004

Paginazione: 178-180

ISSN: 0039-2944

\section{Notizia bibliografica digitale}

Benedetta Papasogli, «Madeleine de Scudéry: une femme de lettres au XVIIe siècle, études réunies par Delphine Denis et Anne Elisabeth Spica», Studi Francesi [Online], 142 (XLVIII | I) | 2004, online dal 30 novembre 2015, consultato il 09 septembre 2021. URL: http://journals.openedition.org/studifrancesi/ 40577 ; DOI: https://doi.org/10.4000/studifrancesi.40577

Questo documento è stato generato automaticamente il 9 septembre 2021.

\section{cc) $($ ) $\ominus$}

Studi Francesi è distribuita con Licenza Creative Commons Attribuzione - Non commerciale - Non opere derivate 4.0 Internazionale. 


\title{
Madeleine de Scudéry: une femme de lettres au XVII siècle, études réunies par Delphine Denis et Anne Elisabeth Spica
}

\author{
Benedetta Papasogli
}

\section{NOTIZIA}

Madeleine de Scudéry: une femme de lettres au XVII siècle, études réunies par DELPHINE DENIS et ANNE ELISABETH SPICA, Artois Presses Université, coll. «Etudes littéraires», 2002, pp. 350.

1 Il convegno di cui questo volume raccoglie gli atti ha celebrato, nel 2001, il tricentenario della morte di Mlle de Scudéry e ha offerto un importante contributo alla messa a fuoco di un'autrice che conosce oggi uno straordinario regain di interesse critico. Lo testimoniano le pagine introduttive di Philippe Sellier, che richiamano le tappe principali della sua recente fortuna, e le conclusioni di Jean Mesnard che suggeriscono direzioni ancora da esplorare pur dopo la raccolta feconda dei saggi qui presentati.

2 Il titolo, privo di ogni accenno alle categorie più sfruttate nel discorso critico su Mlle de Scudéry, come preziosismo o galanteria, annuncia l'estrema apertura dei temi del convegno, e quel carattere di centralità, di punto di convergenza, che la nuova critica riconosce alla scrittrice dopo il radicale ridimensionamento operato in passato da giudizi ingenerosi - si pensi per tutti a quello di Sainte-Beuve. I numerosi contributi si ripartiscono lungo tre assi principali. Il primo punta verso le «Questions de poétique», pur attraversando complessi territori tematici. Centrato sulla poetica del romanzo eroico, che Mlle de Scudéry ha il merito di aver elaborato a partire da testi di teorici italiani del ' 500 , è il contributo inaugurale di Giorgetto Giorgi (Tradition et innovation dans la poétique du roman de Mlle de Scudéry, pp. 29-40); e su problemi di genere insistono 
Marie-Claire Chatelain, che analizza le metamorfosi del modello ovidiano, verso una retorica del sentimento tanto più fine quanto più fondata su valori di sincerità e di naturalezza, in L'héroïde chez Mlle de Scudéry (pp. 41-58), e Rosa Galli Pellegrini, profonda conoscitrice dell'Ibrahim, di cui distingue le storie secondarie in due filoni di «orientales» e di «occidentales» così diversi da configurare due sottogeneri (Thématiques et stratégies narratives des histoires gênoises dans Ibrahim ou l'illustre Bassa, pp. 69-78). Ma già Barbara Piqué, nella sua originale ricognizione dei Cadres allégoriques dans les Conversations de Mlle de Scudéry (pp. 59-68), alle questioni di poetica intreccia le luci dell'analisi morale e le suggestioni dell'immaginario. L'orizzonte morale è vivamente presente nel contributo di Marie Gabrielle Lallemand, La moraliste et la romancière: les trois dernières nouvelles de Mlle de Scudéry (pp. 79-92), volto a mostare come il cambiamento di poetica narrativa nell'opera più tarda della romanziera implichi un mutamento di etica, ispirata ormai alla moderazione e al disincanto; e l'immaginario è evocato nella ricerca di Ch. McCall Probes, Le Pouvoir des sens: une exploration des poésies de Mlle de Scudéry (pp. 93-104) che va scoprendo la ricchezza delle immagini sensoriali nell'opera in versi dell'«illustre Sapho». Chiude questa prima sezione lo studio di Wanda G. Klee e Sabine Koloch che, annunciando i successivi sviluppi del volume in direzione di problemi di ricezione (e infatti le stesse autrici sono presenti nell'ultima parte con un'intervista a Suzanne Weirich, creatrice di un modernissimo «racconto tridimensionale» sulla Carte de Tendre), esplorano uno straordinario caso di dialogo interculturale tra Mlle de Scudéry e il suo ammiratore Wagenseil, che s'interroga con lei sulla bellezza e dignità letteraria della lingua tedesca (Peut-on écrire en allemand? Madeleine de Scudéry et J. C. Wagenseil, un entretien sur la langue allemande, pp. 105-120).

3 La seconda parte porta un titolo denso: La Morale du monde. Questa volta sono focalizzate questioni di morale, colte nell'osservatorio concreto della pratica mondana, intrecciate a miti e stili della «sociabilité» di cui Mlle de Scudéry evidenzia tratti così delicati. Attraverso questa angolatura il contributo di Chantal Morlet-Chantalat su Pythagore et Sapho: réincarnation galante d'un philosophe mythique (pp. 123-132) raggiunge obliquamente lo sfondo dell'antichità che Jean Mesnard, nelle sue conclusioni, indica come soggetto privilegiato di approfondimento per studi futuri su Mlle de Scudéry. I contributi centrali di questa seconda sezione vertono, come ovvio, su una morale del sentimento amoroso, di cui Marie-Odile Sweetser mostra la trasvalutazione in De l'idéal galant à l'héroisme amoureux (pp. 133-140), e i due studi di Carlin e Suzanne Toczyski esplorano i legami con la problematica del matrimonio. In Préciosité et théologie: l'amour conjugal chez Mlle de Scudéry et dans quelques traités catholiques sur le mariage (pp.141-154), è stabilendo un controluce con trattati contempranei di morale coniugale che $\mathrm{C}$. Carlin fa risaltare alcuni tratti centrali dell'etica scuderiana, come l'elogio della tenerezza, dell'amiciza che riequilibria la passione amorosa, l'esigenza della reciprocità. E che l'ideale scuderiano sia tutt'altro che contrario al matrimonio lo dimostrano le arringhe delle sue femmes illustres, quasi tutte sposate, in gran parte vedove, autorizzate di fronte al silenzio del compagno perduto a una presa di parola che comporta un sottile rovesciamento di ruoli nella coppia amorosa: è la tesi di S. Toczyski in Corps sacré, discours souverain: le couple dans Les Femmes illustres (pp. 155-164). Particolare interesse suscita lo studio di Myriam Maître Sapho, «reine» de Tendre: entre monarchie absolue et royauté littéraire (pp. 179-194) ove la fiction del «royaume de Tendre» è esplorata nei suoi risvolti di città ideale, fondata su una dottrina politica rigorosa che coniuga le due grandi definizioni della monarchia moderna, tra signoria e magistratura; e si propone come metafora di quel «particulare» socievole e mondano che s'incuneava, nel Seicento 
francese, con prorompente vitalità fra l'apparato dello Stato assoluto e la «république des Lettres». Chi erano gli abitanti di quello spazio riccamente simbolico, centrato attorno ad una «regina» dal ruolo analogico e antitetico rispetto a quello della monarchia politica? Alain Niderst li passa in rassegna nella sua lettura della corrispondenza che forma il corpus delle Chroniques du Samedi (Pour une édition des Chroniques du Samedi, pp. 195-202). E Anne-Madeleine Goulet ci introduce più da vicino in quel mondo rarefatto e delizioso attraverso un approccio del tutto nuovo: esplorando cioè la funzione della musica nell'universo letterario e mondano di Mlle de Scudéry, autrice lei stessa di versi messi in musica, $\mathrm{e}$ - attraverso le finzioni narrative - nel suo immaginario (Les divertissements musicaux du Samedi, pp.203-217). Alquanto decentrato rispetto a questa seconda sezione, e situato piuttosto nel prolungamento della prima, appare lo studio di Renate Kroll Poésie précieuse, poésie des précieuses: questions de genre et de «gender»(pp. 165-178), contributo prezioso - come sottrarsi al gioco di parole? - per liquidare certe accezioni della nozione di preziosismo banalmente associate al nome della Scudéry, laddove l'idea di galanteria si presta più finemente a individuare gl'idea1i della nostra scrittrice, specie dopo che gli studi di Delphine Denis ne hanno sondato la ricchezza.

4 Vivace e sorprendente è la terza sezione del volume. È dedicata a problemi di ricezione, a prolungamenti e rivisitazioni di un'opera che, rapidamente invecchiata in Francia, ed uscita dalla linea del gusto dominante, ha conosciuto una straordinaria accoglienza in Germania - lo apprendiamo da Volker Kapp, La fortune de Mlle de Scudéry en Allemagne, pp. 219-230, oltre che dai contributi già citati di Klee e Koloch - dove si è creato quasi un mito personale della scrittrice, arbitra dei segreti del cuore, e a lungo il rornanzo eroico è stato preferito alle storie corte e «vere» dell'ultimo Seicento. Questo clima di simpatia contribuisce a spiegare il curioso episodio su cui si sofferma Nathalie Grande in Une Scudéry fantastique: Das Fraulein Von Scudery d'E.T.A. Hoffmann et ses adaptations sur la scène (pp. 292-306): in un racconto di Hoffmann Mlle de Scudéry diviene, da autrice, personaggio, immersa in una trama tenebrosa che fa risaltare in lei il potere quasi sacrale della bontà. Ma anche la linea di una ricezione più prossima, in casa propria, $\mathrm{e}$ addirittura nelle immediate evoluzioni della narrativa secentesca, è esplorata nei bei contributi di Elisa Biancardi, De Madeleine de Scudéry à Mme d'Aulnoy: esthétique galante et merveilleux, pp. 231-240, di Marie-Agnès Thirard, De Madeleine de Scudéry à Mme d'Aulnoy, une réception subversive?, pp. 241-255, e in Shirley Jones Day, Mademoiselle de Scudéry et le roman féminin, pp. 269-278. Complesso e suggestivo appare il rapporto con Mme d'Aulnoy, di cui la Biancardi esplora piuttosto le premesse, scoprendo con finezza le tracce del meraviglioso in rivoli minori del gran fiume dell'opera scuderiana, e la Thirard analizza alcune dinamiche in giochi intertestuali, allusioni, transcodificazioni, presenti nell'opera di Mme d'Aulnoy. Nel contributo della Jones Day, più che un'ottica al fermminile, assai diffusa - e nota - nell'approccio a Mlle de Scudéry, si trova un riscontro puntuale con Mme de La Fayette che nella sua rivoluzione narrativa non manca di profittare di novità introdotte da Mlle de Scudéry, come lo stravolgimento della funzione simbolica dei ruoli maschili e femminili, e la ridefinizione dei rapporti tra l'eroismo e l'amore. Colpisce in questo studio l'uso frequente dell'aggettivo «sensible»: forse già in una prospettiva anticipatrice? Al di là del genere narrativo, Nathalie Pierson si compiace di osservare la trasformazione che temi e situazioni dell'estetica galante subiscono nel passaggio dal romanzo al teatro ( $D u$ romanesque au spectaculaire: Mlle de Scudéry et Quinault, pp. 255-268); e, al di là dei limiti del XVII secolo, uno studio di Françoise Gevrey coglie sottili fili di continuità, magari attraverso 
procedimenti di distanziazione e deformazione burlesca, tra Marivaux et Madeleine de Scudéry (pp. 279-292). Sono solo alcuni sondaggi: ma fanno intravedere un panorama complesso e vivo, e aiutano a cogliere la reale centralità, poco valorizzata da secoli di critica, di «une femme de lettres au XVII ${ }^{\mathrm{e}}$ siècle». 